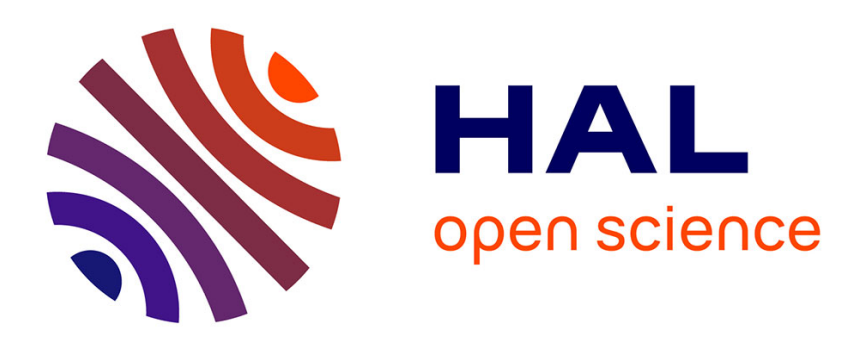

\title{
On complete convergence of triangular arrays of independent random variables
}

István Berkes, Michel Weber

\section{To cite this version:}

István Berkes, Michel Weber. On complete convergence of triangular arrays of independent random variables. Statistics and Probability Letters, 2009, 77 (10), pp.952. 10.1016/j.spl.2006.12.011 . hal00538011

\section{HAL Id: hal-00538011 \\ https://hal.science/hal-00538011}

Submitted on 20 Nov 2010

HAL is a multi-disciplinary open access archive for the deposit and dissemination of scientific research documents, whether they are published or not. The documents may come from teaching and research institutions in France or abroad, or from public or private research centers.
L'archive ouverte pluridisciplinaire HAL, est destinée au dépôt et à la diffusion de documents scientifiques de niveau recherche, publiés ou non, émanant des établissements d'enseignement et de recherche français ou étrangers, des laboratoires publics ou privés. 


\section{Author's Accepted Manuscript}

On complete convergence of triangular arrays of independent random variables

István Berkes, Michel Weber

PII:

S0167-7152(07)00027-2

DOI: doi:10.1016/j.spl.2006.12.011

Reference: STAPRO 4560

To appear in: $\quad$ Statistics \& Probability Letters

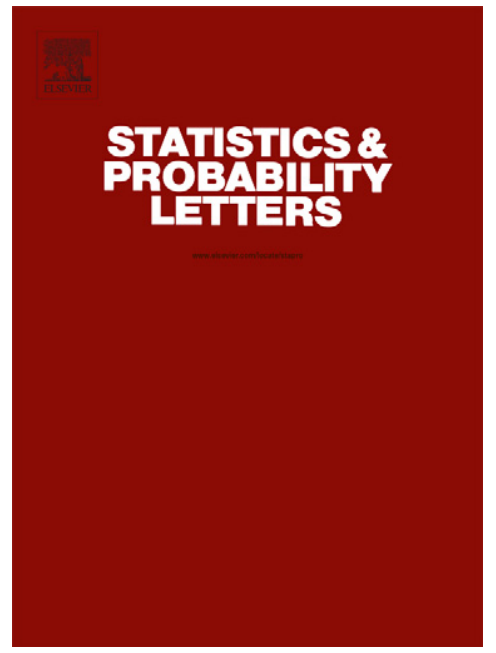

www.elsevier.com/locate/stapro

Received date: 23 March 2006

Revised date: $\quad 11$ September 2006

Accepted date: 19 December 2006

Cite this article as: István Berkes and Michel Weber, On complete convergence of triangular arrays of independent random variables, Statistics \& Probability Letters (2007), doi:10.1016/j.spl.2006.12.011

This is a PDF file of an unedited manuscript that has been accepted for publication. As a service to our customers we are providing this early version of the manuscript. The manuscript will undergo copyediting, typesetting, and review of the resulting galley proof before it is published in its final citable form. Please note that during the production process errors may be discovered which could affect the content, and all legal disclaimers that apply to the journal pertain. 


\title{
On complete convergence of triangular arrays of independent random variables
}

\author{
István BeRKES ${ }^{1}$ and Michel WeBER
}

\begin{abstract}
Given a triangular array $\mathbf{a}=\left\{a_{n, k}, 1 \leq k \leq k_{n}, n \geq 1\right\}$ of positive reals, we study the complete convergence property of $T_{n}=$ $\sum_{k=1}^{k_{n}} a_{n, k} X_{n, k}$ for triangular arrays $\mathcal{X}=\left\{X_{n, k}, 1 \leq k \leq k_{n}, n \geq 1\right\}$ of independent random variables. In the Gaussian case we obtain a simple characterization of density type. Using Skorohod representation and Gaussian randomization, we then derive sufficient criteria for the case when $X_{n, k}$ are in $L^{p}$, and establish a link between the $L^{p}$ case and $L^{2 p}$-case in terms of densities. We finally obtain a density type condition in the case of uniformly bounded random variables.
\end{abstract}

\section{Introduction and results.}

Throughout this paper, we let $\mathcal{X}=\left\{X_{n, k}, 1 \leq k \leq k_{n}, n \geq 1\right\}$ denote a triangular array of real centered independent random variables, and $\mathbf{a}=\left\{a_{n, k}, 1 \leq k \leq k_{n}, n \geq 1\right\}$ with $\left\{k_{n}, n \geq 1\right\}$ non-decreasing, a triangular array of positive reals. When the random variables are symmetric (resp. identically distributed), we will say that the triangular array $\mathcal{X}$ is symmetric (resp. iid). Set, for every $n \geq 1$,

$$
T_{n}=\sum_{k=1}^{k_{n}} a_{n, k} X_{n, k}, \quad A_{n}=\sum_{k=1}^{k_{n}} a_{n, k}, \quad B_{n}^{2}=\sum_{k=1}^{k_{n}} a_{n, k}^{2}, \quad C_{n}=A_{n} / B_{n} .
$$

Let $(\Omega, \mathcal{A}, \mathbf{P})$ be the basic probability space on which $\mathcal{X}$ is defined. Note that $C_{n} \geq 1$. We investigate under what conditions the sequence $T_{n} / A_{n}$ converges completely to $0: T_{n} / A_{n} \stackrel{c . c}{\rightarrow} 0$, which means, as is well-known, that for any $\varepsilon>0$

$$
\sum_{n} \mathbf{P}\left\{\left|T_{n}\right| / A_{n}>\varepsilon\right\}<\infty
$$

The study of this property originates from a well-known paper by Hsu and Robbins (1947) who proved in the case of a single iid sequence $\boldsymbol{\xi}=\left\{\xi, \xi_{n}, n \geq 1\right\}$ with partial sums $S_{n}=$ $\sum_{k=1}^{n} \xi_{k}, n=1,2, \ldots$ that $\mathbf{E} \xi=0, \mathbf{E} \xi^{2}<\infty$ imply $S_{n} / n \stackrel{\text { c.c. }}{\rightarrow} 0$. Shortly afterward, Erdős (1949) proved the validity of the converse implication. Since then, the study of various possible generalizations of this result (subsequence case, the theorems of Baum-Katz (1965), extensions to

1 Research supported by Hungarian National Foundation for Scientific Research, Grants T $043037, \mathrm{~T} 037886$ and $\mathrm{K} 61052$

AMS Subject Classification 2000: Primary 60G50, 60F15, 60B12, Secondary 60G15.

Keywords: Complete convergence, triangular arrays, independent random variables. 
triangular arrays of independent random variables, Banach space valued random variables) have received a lot of attention. See, for example, the works of Pruitt (1966), Rohatgi (1971), Fazekas (1985, 1992), Hu-Móricz-Taylor (1989), Kuczmaszewska-Szynal (1988, 1990, 1994), Gut (1992), Li-Rao-Wang (1992), Rao-Wang-Yang (1993), Sung (1997), Adler-Cabrera-Rosalsky-Volodin (1999), Hu-Rosalsky-Szynal-Volodin (1999), Ahmed-Antonini-Volodin (2002). The purpose of the present paper is to present new necessary as well as sufficient criteria for the complete convergence of triangular arrays of independent random variables, and discuss their relations with known results in the literature.

We start our investigations with the Gaussian case, because of the classical Gaussian randomization procedure for sums of independent random variables, and also because this case is in general very informative. If $\mathcal{X}$ is Gaussian, the problem can be simply settled. Put

$$
\mathcal{L}(\mathbf{a})=\limsup _{x \rightarrow \infty} \frac{\log \sharp\left\{n: C_{n} \leq x\right\}}{x^{2}} .
$$

Then we have the following characterization.

Theorem 1. Assume that the $X_{n, k}$ are i.i.d. standard Gaussian variables. Then we have

$$
T_{n} / A_{n} \stackrel{\text { c.c. }}{\longrightarrow} 0 \quad \Longleftrightarrow \quad \mathcal{L}(\mathbf{a})=0 .
$$

In view of this complete result, it is natural to attack the general i.i.d. case using invariance principles. Applying Skorohod embedding for the row sums of the triangular array $\mathcal{X}$ leads, under natural conditions on the stopping times in the Skorohod representation, to a necessary and sufficient criterion for $T_{n} / A_{n} \stackrel{\text { c.c. }}{\longrightarrow} 0$, see Proposition 10 . This condition, in turn, leads to sufficient criteria under the existence of higher moments. In particular, we will prove

Theorem 2. Assume that $\mathbf{E} X_{n, k}^{2}=1$ and $X_{n, k} \in L^{2 p}$ for some $p \geq 2$. Then the relation

$$
\sum_{n} \frac{\left(\sum_{k=1}^{k_{n}} a_{n, k}^{4}\right)^{p / 2}}{\left(\sum_{k=1}^{k_{n}} a_{n, k}^{2}\right)^{p}}<\infty
$$

implies $T_{n} / A_{n} \stackrel{\text { c.c. }}{\longrightarrow} 0$.

To compare this result with the Gaussian case, note that $\mathcal{L}(\mathbf{a})=0$ is equivalent to

$$
\sum_{n} \exp \left(-\delta \frac{\left(\sum_{k=1}^{k_{n}} a_{n, k}\right)^{2}}{\sum_{k=1}^{k_{n}} a_{n, k}^{2}}\right)<\infty \quad \text { for all } \delta>0 \text {. }
$$

In the case when $\mathcal{X}$ is also symmetric, the condition in Theorem 2 can be weakened.

Theorem 3. Assume that $\mathcal{X}$ is symmetric, $\mathbf{E} X_{n, k}^{2}=1$ and $X_{n, k} \in L^{2 p}$ for some $p \geq 2$. Then the relation

$$
\sum_{n} \frac{\left(\sum_{k=1}^{k_{n}} a_{n, k}^{4}\right)^{p / 2}}{\left(\sum_{k=1}^{k_{n}} a_{n, k}\right)^{2 p} \log ^{p} n}<\infty
$$

implies $T_{n} / A_{n} \stackrel{\text { c.c. }}{\longrightarrow} 0$. 
Recall that the array $\mathcal{X}$ is stochastically bounded by a random variable $X$ if there is a constant $D$ such that $\mathbf{P}\left\{\left|X_{n, k}\right|>x\right\} \leq D \mathbf{P}\{|D X|>x\}$ for all $x>0$ and for all $n \geq 1$, $1 \leq k \leq k_{n}$. We will prove the following result.

THEOREM 4. Let $\mathcal{X}$ be a symmetric triangular array stochastically bounded by a square integrable random variable $X$. Assume that for any $\varepsilon>0$,

(a) $\sum_{1 \leq k \leq l<\infty} \mathbf{P}\left\{|X| \geq \varepsilon A_{l} / a_{k}\right\}<\infty$.

Further assume that for some integer $r \geq 2$ and any $\varepsilon>0$,

(b) $\quad \sum_{n \geq 1} \mathbf{P}\left\{\left|T_{n}\right|>\varepsilon A_{n}\right\}^{r}<\infty$.

Then

(c) $T_{n} / A_{n} \stackrel{\text { c.c. }}{\longrightarrow} 0$.

Conversely, if the triangular array $\mathcal{X}$ is iid symmetric, then (c) implies (a).

The next result concerns the uniformly bounded case. We show that a condition similar to that assumed in the Gaussian case suffices for complete convergence. Put, for any positive integer $n$,

$$
V_{n}^{2}=\sum_{k=1}^{k_{n}} a_{n, k}^{2} X_{n, k}^{2} .
$$

TheOREM 5. Let $\mathcal{X}$ be a triangular array of real centered, uniformly bounded independent random variables. Assume that for any $\varepsilon>0$

$$
\mathbf{E} \sup _{m \geq 1} \frac{\sharp\left\{n: m<A_{n} / V_{n} \leq m+1\right\}}{\exp \left\{\varepsilon m^{2}\right\}}<\infty .
$$

Then $T_{n} / A_{n} \stackrel{\text { c.c. }}{\longrightarrow} 0$.

Our final result establishes a link between the complete convergence of arrays in the $L^{p}$ and $L^{2 p}$-case. Remarkably, the link is provided by the density condition in the Gaussian case in Theorem 1. We need a preliminary definition.

Definition. Let $p \geq 2$. We say that $\mathbf{a}$ is p-regular if any triangular array $\mathcal{X}$ of real centered iid random variables with finite pth moment satisfies $T_{n} / A_{n} \stackrel{\text { c.c. }}{\rightarrow} 0$.

Let $\mathbf{a}$ be a triangular array of positive reals. Define

$$
\mathbf{a}^{2}:=\left\{a_{n, k}^{2}, 1 \leq k \leq k_{n}, n \geq 1\right\} .
$$

Then we have

TheOREM 6. Let $p \geq 2$ and assume that $\mathbf{a}^{2}$ is p-regular. Then $\mathbf{a}$ is $2 p$-regular iff $\mathcal{L}(\mathbf{a})=0$.

\section{Proofs.}

Proof of Theorem 1. Before giving the proof, recall for the reader's convenience an elementary estimate for Gaussian random variables due to Komatsu-Pollak (see Mitrinović (1970), p. 178). 
Lemma 7. The Mills's ratio $R(x)=e^{x^{2} / 2} \int_{x}^{\infty} e^{-t^{2} / 2} d t$ satisfies

$$
\frac{2}{\sqrt{x^{2}+4}+x} \leq R(x) \leq \frac{2}{\sqrt{x^{2}+\frac{8}{\pi}}+x} \leq \sqrt{\frac{\pi}{2}} \quad \text { for all } x>0 .
$$

Note that

$$
\mathbf{P}\left\{\left|T_{n}\right| / A_{n}>\varepsilon\right\}=\mathbf{P}\left\{|\mathcal{N}(0,1)|>\varepsilon C_{n}\right\} \asymp \frac{1}{1+\varepsilon C_{n}} e^{-\left(\varepsilon C_{n}\right)^{2} / 2} \quad \text { as } n \rightarrow \infty,
$$

where the symbol $\asymp$ means that the ratio of the two sides is between positive constants. Thus it follows that $T_{n} / A_{n} \stackrel{\text { c.c. }}{\longrightarrow} 0$ if and only if the series

$$
\sum_{n} e^{-\delta C_{n}^{2}}
$$

converges for any $\delta>0$. And this is equivalent to $\mathcal{L}(\mathbf{a})=0$ (for a proof, see e.g. Weber (1995), pp. 402-403).

Proof of Theorem 6. The proof relies upon several intermediate results. Let $\boldsymbol{\xi}=\left\{\xi_{k}, k \geq 1\right\}$ be a sequence of real centered independent square integrable random variables defined on the probability space $(\Omega, \mathcal{A}, \mathbf{P})$, and let $\mathbf{w}=\left\{w_{k}, k \geq 1\right\}$ be a sequence of positive reals. Put, for any positive integer $m$,

$$
S_{m}=\sum_{k=1}^{m} w_{k} \xi_{k}, \quad W_{m}=\sum_{k=1}^{m} w_{k}, \quad M_{m}=\sum_{k=1}^{m} w_{k}^{2} .
$$

Recall the Skorokhod embedding scheme (see e.g. Breiman (1968)): there exists, after suitably enlarging the probability space, a linear Brownian motion $\mathcal{B}=\{B(t), 0 \leq t<\infty\}$ starting at 0 , and a sequence $\tau_{1}, \tau_{2}, \ldots$ of independent non-negative random variables with $\mathbf{E} \tau_{k}=w_{k}^{2} \mathbf{E} \xi_{k}^{2}$, $k \geq 1$ such that, with $\tau_{0}=0$ a.s.,

$$
\left\{B\left(\sum_{j=0}^{k} \tau_{j}\right)-B\left(\sum_{j=0}^{k-1} \tau_{j}\right), k \geq 1\right\} \stackrel{\mathcal{D}}{=}\left\{w_{k} \xi_{k}, k \geq 1\right\}
$$

Put, for any real $x$,

$$
\Psi(x)=\frac{1}{\sqrt{2 \pi}} \int_{x}^{\infty} e^{-u^{2} / 2} d u
$$

Lemma 8. Let $\varepsilon, h, \delta$ be positive numbers with $\varepsilon>h>\sqrt{2 \delta}$, and put

$$
\Delta_{m}=\Delta_{m}(\delta)=\mathbf{P}\left\{\left|\sum_{j=0}^{m} \tau_{j}-M_{m}\right| \geq \delta M_{m}\right\}
$$

Then, for any positive integer $m$, we have

$\Psi\left((\varepsilon+h) \frac{W_{m}}{\sqrt{M_{m}}}\right)-4 \Psi\left(\frac{h W_{m}}{\sqrt{2 \delta M_{m}}}\right)-\Delta_{m} \leq \mathbf{P}\left\{\left|S_{m}\right|>\varepsilon W_{m}\right\} \leq \Psi\left((\varepsilon-h) \frac{W_{m}}{\sqrt{M_{m}}}\right)+4 \Psi\left(\frac{h W_{m}}{\sqrt{2 \delta M_{m}}}\right)+\Delta_{m}$. 
Proof. We observe that

$$
\begin{aligned}
& \mathbf{P}\left\{\left|S_{m}\right|>\varepsilon W_{m}\right\}=\mathbf{P}\left\{\left|B\left(\sum_{j=0}^{m} \tau_{j}\right)\right|>\varepsilon W_{m}\right\} \\
& \leq \mathbf{P}\left\{\left|B\left(M_{m}\right)\right|>(\varepsilon-h) W_{m}\right\}+\mathbf{P}\left\{\left|B\left(\sum_{j=0}^{m} \tau_{j}\right)\right|>\varepsilon W_{m},\left|B\left(M_{m}\right)\right| \leq(\varepsilon-h) W_{m}\right\} \\
& \leq \Psi\left(\frac{(\varepsilon-h) W_{m}}{\sqrt{M_{m}}}\right)+\mathbf{P}\left\{\left|B\left(\sum_{j=0}^{m} \tau_{j}\right)-B\left(M_{m}\right)\right| \geq h W_{m}\right\} \\
& \leq \Psi\left(\frac{(\varepsilon-h) W_{m}}{\sqrt{M_{m}}}\right)+\mathbf{P}\left\{\left|\sum_{j=0}^{m} \tau_{j}-M_{m}\right| \geq \delta M_{m}\right\}+\mathbf{P}\left\{\sup _{|\theta-1| \leq \delta}\left|B\left(\theta M_{m}\right)-B\left(M_{m}\right)\right| \geq h W_{m}\right\} \\
& =\Psi\left(\frac{(\varepsilon-h) W_{m}}{\sqrt{M_{m}}}\right)+\mathbf{P}\left\{\left|\sum_{j=0}^{m} \tau_{j}-M_{m}\right| \geq \delta M_{m}\right\}+\mathbf{P}\left\{\sup _{|\theta-1| \leq \delta}|B(\theta)-B(1)| \geq h \frac{W_{m}}{\sqrt{M_{m}}}\right\} .
\end{aligned}
$$

Conversely,

$$
\begin{aligned}
& \Psi\left((\varepsilon+h) \frac{W_{m}}{\sqrt{M_{m}}}\right)=\mathbf{P}\left\{\left|B\left(M_{m}\right)\right|>(\varepsilon+h) W_{m}\right\} \\
& \leq \mathbf{P}\left\{\left|B\left(\sum_{j=0}^{m} \tau_{j}\right)\right|>\varepsilon W_{m}\right\}+\mathbf{P}\left\{\left|B\left(M_{m}\right)\right|>(\varepsilon+h) W_{m},\left|B\left(\sum_{j=0}^{m} \tau_{j}\right)\right| \leq \varepsilon W_{m}\right\} \\
& \leq \mathbf{P}\left\{\left|B\left(\sum_{j=0}^{m} \tau_{j}\right)\right|>\varepsilon W_{m}\right\}+\mathbf{P}\left\{\left|B\left(\sum_{j=0}^{m} \tau_{j}\right)-B\left(M_{m}\right)\right| \geq h W_{m}\right\} \\
& \leq \mathbf{P}\left\{\left|B\left(\sum_{j=0}^{m} \tau_{j}\right)\right|>\varepsilon W_{m}\right\}+\mathbf{P}\left\{\left|\sum_{j=0}^{m} \tau_{j}-M_{m}\right| \geq \delta M_{m}\right\} \\
& +\mathbf{P}\left\{\sup _{|\theta-1| \leq \delta}\left|B\left(\theta M_{m}\right)-B\left(M_{m}\right)\right| \geq h W_{m}\right\} \\
& =\mathbf{P}\left\{\left|B\left(\sum_{j=0}^{m} \tau_{j}\right)\right|>\varepsilon W_{m}\right\}+\mathbf{P}\left\{\left|\sum_{j=0}^{m} \tau_{j}-M_{m}\right| \geq \delta M_{m}\right\}+\mathbf{P}\left\{\sup _{|\theta-1| \leq \delta}|B(\theta)-B(1)| \geq h \frac{W_{m}}{\sqrt{M_{m}}}\right\} .
\end{aligned}
$$

As $B$ has stationary increments, we get by using scale invariance, the symmetry of the law of $B$ and Eq. 1.5.1 p. 43 in Csörgö and Révész (1981),

$$
\begin{aligned}
\mathbf{P}\left\{\sup _{|\theta-1| \leq \delta}|B(\theta)-B(1)| \geq h \frac{W_{m}}{\sqrt{M_{m}}}\right\} & =\mathbf{P}\left\{\sup _{u \in[0,2 \delta]}|B(u)| \geq \frac{h W_{m}}{\sqrt{M_{m}}}\right\} \\
& =\mathbf{P}\left\{\sup _{0 \leq u \leq 1}|B(u)| \geq \frac{h W_{m}}{\sqrt{2 \delta M_{m}}}\right\} \\
& =\mathbf{P}\left\{\max \left(\sup _{0 \leq u \leq 1} B(u), \sup _{0 \leq u \leq 1}-B(u)\right) \geq \frac{h W_{m}}{\sqrt{2 \delta M_{m}}}\right\} \\
& \leq 2 \mathbf{P}\left\{\sup _{0 \leq u \leq 1} B(u) \geq \frac{h W_{m}}{\sqrt{2 \delta M_{m}}}\right\}=4 \Psi\left(\frac{h W_{m}}{\sqrt{2 \delta M_{m}}}\right) .
\end{aligned}
$$

Consequently

$\Psi\left((\varepsilon+h) \frac{W_{m}}{\sqrt{M_{m}}}\right)-4 \Psi\left(\frac{h W_{m}}{\sqrt{2 \delta M_{m}}}\right)-\Delta_{m} \leq \mathbf{P}\left\{\left|S_{m}\right|>\varepsilon W_{m}\right\} \leq \Psi\left((\varepsilon-h) \frac{W_{m}}{\sqrt{M_{m}}}\right)+4 \Psi\left(\frac{h W_{m}}{\sqrt{2 \delta M_{m}}}\right)+\Delta_{m}$.

This completes the proof. 
We shall apply Lemma 8 to triangular arrays. Let again $\mathcal{X}=\left\{X_{n, k}, 1 \leq k \leq k_{n}, n \geq 1\right\}$ be a triangular array of real centered independent random variables and $\mathbf{a}=\left\{a_{n, k}, 1 \leq k \leq\right.$ $\left.k_{n}, n \geq 1\right\}$ a triangular array of positive reals. By considering, if necessary, a larger probability space, we can always assume that there exists a sequence $\boldsymbol{\xi}^{1}, \boldsymbol{\xi}^{2}, \ldots$ such that for each positive integer $n$,

$$
\boldsymbol{\xi}^{n}=\left\{\xi_{n, k}, k \geq 1\right\} \quad \text { with } \quad \xi_{n, k}=X_{n, k}, 1 \leq k \leq k_{n},
$$

and $\boldsymbol{\xi}^{n}$ is a sequence of independent random variables. Further the sequences $\boldsymbol{\xi}^{1}, \boldsymbol{\xi}^{2}, \ldots$ are mutually independent. By suitably enlarging the probability space, there exists for each integer $n$ a linear Brownian motion $\mathcal{B}^{n}=\left\{B^{n}(t), 0 \leq t<\infty\right\}$ starting at 0 and a sequence $\tau_{1}^{n}, \tau_{2}^{n}, \ldots$ of independent non-negative random variables with $\mathbf{E} \tau_{k}^{n}=a_{n, k}^{2} \mathbf{E} \xi_{n, k}^{2}, k \geq 1$ such that, with $\tau_{0}^{n}=0$ a.s.,

$$
\left\{B^{n}\left(\sum_{j=0}^{k} \tau_{j}^{n}\right)-B^{n}\left(\sum_{j=0}^{k-1} \tau_{j}^{n}\right), k \geq 1\right\} \stackrel{\mathcal{D}}{=}\left\{a_{n, k} \xi_{n, k}, k \geq 1\right\} .
$$

In fact, in each step, it would be enough to let $k$ run between 1 and $k_{n}$. By applying Lemma 8 with the choice $\boldsymbol{\xi}=\boldsymbol{\xi}^{n}, m=k_{n}$, we now easily deduce the following corollary.

Corollary 9. Let $\varepsilon, h, \delta$ be positive reals with $\varepsilon>h>\sqrt{2 \delta}$. Then, with the notation (1), for $n=1,2, \ldots$

$\Psi\left((\varepsilon+h) C_{n}\right)-4 \Psi\left(\frac{h}{\sqrt{2 \delta}} C_{n}\right)-\Delta_{n}(\delta) \leq \mathbf{P}\left\{\left|T_{n}\right|>\varepsilon A_{n}\right\} \leq \Psi\left((\varepsilon-h) C_{n}\right)+4 \Psi\left(\frac{h}{\sqrt{2 \delta}} C_{n}\right)+\Delta_{n}(\delta)$,

where

$$
\Delta_{n}(\delta)=\mathbf{P}\left\{\left|\sum_{j=0}^{k_{n}} \tau_{j}^{n}-B_{n}^{2}\right| \geq \delta B_{n}^{2}\right\} .
$$

This result will allow us to establish the following statement.

Proposition 10. Assume that $\mathcal{X}$ and a satisfy

$$
\sum_{m} \Delta_{m}(\delta)<\infty, \quad \text { for all } \delta>0
$$

Then

$$
T_{n} / A_{n} \stackrel{\text { c.c. }}{\longrightarrow} 0 \quad \Longleftrightarrow \quad \mathcal{L}(\mathbf{a})=0
$$

This proposition can be viewed as an extension of Theorem 1, since in the Gaussian case $\tau_{j}^{n} \stackrel{a . s .}{=} a_{n, j}^{2}$.

Proof. The key lies in the comparison between $\Psi\left((\varepsilon+h) C_{n}\right)$ and $\Psi\left(\frac{h}{\sqrt{2 \delta}} C_{n}\right)$, which is achieved by using Lemma 7. The implication $\mathcal{L}(\mathbf{a})=0 \Rightarrow T_{n} / A_{n} \stackrel{\text { c.c. }}{\longrightarrow} 0$ is easy. Indeed, if $\mathcal{L}(\mathbf{a})=0$, then for any $\rho>0$ the series $\sum_{n} e^{-\rho C_{n}^{2}}$ converges, or equivalently,

$$
\sum_{n} \Psi\left(\rho C_{n}\right)<\infty \quad \text { for all } \rho>0 .
$$

Let $\varepsilon>0$, and choose $h, \delta$ in Corollary 9 such that $h=\varepsilon / 2>\sqrt{2 \delta}$. By Corollary 9 and the assumption made, the series $\sum_{n} \mathbf{P}\left\{\left|T_{n}\right|>\varepsilon A_{n}\right\}$ converges provided

$$
\sum_{n} \Psi\left((\varepsilon-h) C_{n}\right)<\infty, \quad \sum_{n} \Psi\left(\frac{h}{\sqrt{2 \delta}} C_{n}\right)<\infty .
$$


And this holds true if $\sum_{n} \Psi\left((\varepsilon / 2) C_{n}\right)<\infty$, which is satisfied by assumption. Hence the first part of Proposition 10 is proved.

Conversely, if $T_{n} / A_{n} \stackrel{c . c .}{\rightarrow} 0$, then the series $\sum_{n} \mathbf{P}\left\{\left|T_{n}\right|>\varepsilon A_{n}\right\}$ converges for any $\varepsilon>0$. We shall prove that (3) holds true. We distinguish two cases.

Case 1: $\liminf _{n \rightarrow \infty} C_{n}=\infty$. Let $\rho>0$ be fixed, we choose $\varepsilon, h, \delta$ such as $\varepsilon=h=\rho / 2, \delta=1 / 8$, so that $\frac{h}{\sqrt{2 \delta}}=2 \rho$. Then $\Psi\left((\varepsilon+h) C_{n}\right)=\Psi\left(\rho C_{n}\right)$ and $\Psi\left(\frac{h}{\sqrt{2 \delta}} C_{n}\right)=\Psi\left(2 \rho C_{n}\right)$. By Lemma 7 ,

$$
\Psi\left(\rho C_{n}\right) \asymp \frac{1}{1+\rho C_{n}} e^{-\left(\rho C_{n}\right)^{2} / 2}, \quad \Psi\left(2 \rho C_{n}\right) \asymp \frac{1}{1+2 \rho C_{n}} e^{-2\left(\rho C_{n}\right)^{2}},
$$

so that, for any $\rho<\rho_{1}<\rho_{2}<2 \rho$, if $n$ is sufficiently large

$$
\Psi\left(\rho C_{n}\right) \geq e^{-\left(\rho_{1} C_{n}\right)^{2} / 2}, \quad 4 \Psi\left(2 \rho C_{n}\right) \leq e^{-\left(\rho_{2} C_{n}\right)^{2} / 2} .
$$

Therefore

$$
\Psi\left(\rho C_{n}\right)-4 \Psi\left(2 \rho C_{n}\right) \geq e^{-\left(\rho_{1} C_{n}\right)^{2} / 2}\left(1-e^{-\left(\rho_{2}^{2}-\rho_{1}^{2}\right)\left(C_{n}\right)^{2} / 2}\right) \geq(1 / 2) e^{-\left(\rho_{1} C_{n}\right)^{2} / 2},
$$

for $n$ sufficiently large. In view of Corollary 9, and assumption (2) this implies that the series $\sum_{n} e^{-\left(\rho_{1} C_{n}\right)^{2} / 2}$ converges. This being true for any $\rho>0$ and any $\rho_{1}>\rho$, it follows that $(3)$ is satisfied, as claimed.

Case 2: $\liminf _{n \rightarrow \infty} C_{n}<\infty$. In this case there exist a sequence of indices $\left\{n_{j}, j \geq 1\right\}$ and a real $t$ such that $\lim _{j \rightarrow \infty} C_{n_{j}}=t$. Choose $\rho>0$ such that $\Psi(\rho t)>4 \Psi(2 \rho t)$, and let again $\varepsilon, h, \delta$ such as $\varepsilon=h=\rho / 2, \delta=\frac{1}{8}$. Applying Corollary 9 for $n=n_{j}, j=1,2, \ldots$ gives

$$
\Psi\left(\rho C_{n_{j}}\right)-4 \Psi\left(2 \rho C_{n_{j}}\right) \leq \mathbf{P}\left\{\left|T_{n_{j}}\right|>\varepsilon A_{n_{j}}\right\}+\Delta_{n_{j}}(\delta) .
$$

Letting now $j$ tend to infinity implies

$$
0<\Psi(\rho t)-4 \Psi(2 \rho t) \leq \liminf _{j \rightarrow \infty}\left(\mathbf{P}\left\{\left|T_{n_{j}}\right|>\varepsilon A_{n_{j}}\right\}+\Delta_{n_{j}}(\delta)\right),
$$

which contradicts the fact that both series $\sum_{n} \mathbf{P}\left\{\left|T_{n}\right|>\varepsilon A_{n}\right\}, \sum_{n} \Delta_{n}(\delta)$ converge. The proof is now complete.

We can now pass to the proof of Theorem 6 . Let $p \geq 2$ and let $\mathbf{a}=\left\{a_{n, k}, 1 \leq k \leq k_{n}, n \geq 1\right\}$ be a triangular array of positive reals such that $\mathbf{b}=\mathbf{a}^{2}$ is $p$-regular. Let $\mathcal{X}=\left\{X_{n, k}, 1 \leq k \leq\right.$ $\left.k_{n}, n \geq 1\right\}$ be a triangular array of real centered iid random variables with finite $2 p$-th moment. We shall make use of the fact (Fisher (1992), Theorem 2.1) that for each $n$, we can assume that $\left\{\tau_{k}^{n}, 1 \leq k \leq k_{n}\right\} \stackrel{\mathcal{D}}{=}\left\{a_{n, k}^{2} \theta_{k}^{n}, 1 \leq k \leq k_{n}\right\}$, and $\left\{\theta_{k}^{n}, 1 \leq k \leq k_{n}\right\}$ is an iid sequence with finite $p$-th moment. As $\mathbf{b}$ is $p$-regular, (2) is satisfied. Using Proposition 10, we get the desired conclusion.

Remark. Although the characterization given in Theorem 6 is simple, it is rather abstract. Usually condition (2) is as difficult to check as the fact that $\mathbf{a}$ is $2 p$-regular. Thus the interest in a statement like Theorem 6 is the link established between $p$-regularity and $2 p$-regularity, via the arrays $\mathbf{a}$ and $\mathbf{b}$.

It is possible to check directly condition (2), by imposing conditions on the weights, which, however, appear to be stronger than the condition $\mathcal{L}(\mathbf{a})=0$. To see this, we shall use some 
arguments from Weber (2006). In order to avoid unnecessarily heavy notation, we simply return to the setting considered in Lemma 8 , and will bound the quantity

$$
\Delta_{m}=\Delta_{m}(\delta)=\mathbf{P}\left\{\left|\sum_{j=0}^{m} \tau_{j}-M_{m}\right| \geq \delta M_{m}\right\} .
$$

Using inequality (1.2) in Davis (1976) we see that if $\mathbf{E}\left|\xi_{i}\right|^{2+\varepsilon}<\infty$ for some $\varepsilon>0$, the sequence of stopping times $\tau_{i}$ satisfies

$$
\mathbf{E} \tau_{i}^{1+\varepsilon / 2} \leq C w_{i}^{2+\varepsilon} \mathbf{E}\left|\xi_{i}\right|^{2+\varepsilon},
$$

where the constant $C$ depends on $\varepsilon$ only. Let $p \geq 2$. Assume that for any positive integer $j$, $\xi_{i} \in L^{2 p}$, and moreover

$$
Q_{p}(\boldsymbol{\xi}):=\sup _{j \geq 1}\left\|\xi_{i}\right\|_{p}<\infty
$$

Put for any positive integer $l$,

$$
x_{l}=\tau_{l}-\mathbf{E} \tau_{l}=\tau_{l}-w_{l}^{2} .
$$

Then using (4) with $2(p-1)=\varepsilon$ gives

$$
\mathbf{E}\left|x_{l}\right|^{p} \leq 2^{p}\left(\mathbf{E}\left|\tau_{l}\right|^{p}+w_{l}^{2 p}\right) \leq C_{p}^{\prime}\left(1+Q_{p}^{p}(\boldsymbol{\xi})\right) w_{l}^{2 p}
$$

where $C_{p}^{\prime}$ depends on $p$ only, and may vary in the next lines. Further note that in the case $\xi_{l} \in L^{4}, l \geq 1$ we have

$$
0 \leq \mathbf{E} x_{l}^{2}=\mathbf{E} \tau_{l}^{2}-\left(\mathbf{E} \tau_{l}\right)^{2} \leq \mathbf{E} \tau_{l}^{2} \leq C_{2}^{\prime} w_{l}^{4} \mathbf{E}\left|\xi_{l}\right|^{4} .
$$

Apply now Rosenthal's inequality (see e.g. Petrov (1995), p. 59). In view of centering and independence of the $x_{l}$ 's, we get

$$
\begin{aligned}
\mathbf{E}\left|\sum_{l=1}^{m}\left(\tau_{l}-w_{l}^{2}\right)\right|^{p} & \leq C_{p}^{\prime}\left(\sum_{l=1}^{m} w_{l}^{2 p}+\left(\sum_{l=1}^{m} \mathbf{E} x_{l}^{2}\right)^{p / 2}\right) \\
& \leq C_{p}^{\prime}\left(1+Q_{p}^{p}(\boldsymbol{\xi})\right)\left(\sum_{l=1}^{m} w_{l}^{2 p}+\left(\sum_{l=1}^{m} w_{l}^{4}\right)^{p / 2}\right) \leq C_{p}^{\prime}\left(1+Q_{p}^{p}(\boldsymbol{\xi})\right)\left(\sum_{l=1}^{m} w_{l}^{4}\right)^{p / 2} .
\end{aligned}
$$

Consequently, by using Chebyshev's inequality,

$$
\Delta_{m}(\delta)=\mathbf{P}\left\{\left|\sum_{j=0}^{m} \tau_{j}-M_{m}\right| \geq \delta M_{m}\right\} \leq C_{p}^{\prime}\left(1+Q_{p}^{p}(\boldsymbol{\xi})\right)\left(\frac{\sum_{l=1}^{m} w_{l}^{4}}{\left(\delta M_{m}\right)^{2}}\right)^{p / 2} .
$$

We thus see that condition (2) holds provided

$$
\sum_{m}\left(\frac{\left[\sum_{l=1}^{m} w_{l}^{4}\right]^{1 / 2}}{M_{m}}\right)^{p}<\infty
$$

For triangular arrays, this means that

$$
\sum_{n}\left(\frac{\left[\sum_{k=1}^{k_{n}} a_{n, k}^{4}\right]^{1 / 2}}{\sum_{k=1}^{k_{n}} a_{n, k}^{2}}\right)^{p}<\infty
$$


establishing Theorem 2. As we noted earlier, $\mathcal{L}(\mathbf{a})=0$ is equivalent to

$$
\sum_{n} \exp \left(-\delta \frac{\left(\sum_{k=1}^{k_{n}} a_{n, k}\right)^{2}}{\sum_{k=1}^{k_{n}} a_{n, k}^{2}}\right)<\infty \quad \text { for all } \delta>0 .
$$

Proof of Theorem 3. Since $\mathcal{X}$ is symmetric, it has the same law as $\mathcal{X}=\left\{\varepsilon_{n, k} X_{n, k}, 1 \leq k \leq\right.$ $\left.k_{n}, n \geq 1\right\}$, where $\varepsilon=\left\{\varepsilon_{n, k}, 1 \leq k \leq k_{n}, n \geq 1\right\}$ is a Rademacher sequence defined on a joint probability space $\left(\Omega_{\varepsilon}, \mathcal{A}_{\varepsilon}, \mathbf{P}_{\varepsilon}\right)$ (with corresponding expectation symbol $\left.\mathbf{E}_{\varepsilon}\right)$. Put

$$
Y_{n}=\sum_{k=1}^{k_{n}} a_{n, k} \varepsilon_{n, k} X_{n, k}, \quad Q_{n}=\frac{\sum_{k=1}^{k_{n}} a_{n, k}^{2} X_{n, k}^{2}}{B_{n}^{2}} .
$$

Let $\left\{\Omega_{n}, n \geq 1\right\}$ be a sequence of positive reals. Write

$$
\mathbf{P}\left\{\frac{\left|T_{n}\right|}{A_{n}}>\varepsilon\right\}=\mathbf{E} \mathbf{P}_{\varepsilon}\left\{\frac{\left|Y_{n}\right|}{A_{n}}>\varepsilon\right\} \leq \mathbf{P}\left\{Q_{n}>\Omega_{n}\right\}+\mathbf{E} \mathbf{1}_{\left\{Q_{n} \leq \Omega_{n}\right\}} \mathbf{P}_{\varepsilon}\left\{\frac{\left|Y_{n}\right|}{A_{n}}>\varepsilon\right\} .
$$

Further, there exists an absolute constant $C$ such that

$$
\mathbf{P}_{\varepsilon}\left\{\frac{\left|Y_{n}\right|}{A_{n}}>\varepsilon\right\} \leq \exp \left\{-C \frac{\varepsilon^{2} A_{n}^{2}}{\sum_{k=1}^{k_{n}} a_{n, k}^{2} X_{n, k}^{2}}\right\}=\exp \left\{-C \frac{\varepsilon^{2} A_{n}^{2}}{Q_{n} B_{n}^{2}}\right\}=\exp \left\{-C \frac{\varepsilon^{2} C_{n}^{2}}{Q_{n}}\right\} .
$$

We deduce that

$$
\mathbf{P}\left\{\frac{\left|T_{n}\right|}{A_{n}}>\varepsilon\right\} \leq \mathbf{P}\left\{Q_{n}>\Omega_{n}\right\}+\exp \left\{-C \frac{\varepsilon^{2} C_{n}^{2}}{\Omega_{n}}\right\}
$$

It follows that if

$$
\text { a) } \sum_{n=1}^{\infty} \mathbf{P}\left\{Q_{n}>\Omega_{n}\right\}<\infty, \quad \text { b) } \sum_{n=1}^{\infty} \exp \left\{-C \frac{\varepsilon^{2} C_{n}^{2}}{\Omega_{n}}\right\}<\infty .
$$

then $T_{n} / A_{n} \stackrel{\text { c.c. }}{\longrightarrow} 0$. Choosing in particular (with $L>1$ )

$$
\Omega_{n}=C_{n}^{2} /(L \log n)
$$

shows that $T_{n} / A_{n} \stackrel{\text { c.c. }}{\longrightarrow} 0$, provided that

$$
\sum_{n=1}^{\infty} \mathbf{P}\left\{Q_{n}>\lambda C_{n}^{2} / \log n\right\}<\infty
$$

for any $\lambda>0$. To connect the last sum with the sum in Theorem 3, we use Rosenthal's inequality. Recall that we assumed for $1 \leq k \leq k_{n}, n \geq 1$ that $\mathbf{E} X_{n, k}^{2}=1$, and for some $p \geq 2$, $X_{n, k} \in L^{2 p}$. Put

$$
Y_{n, k}=a_{n, k}^{2}\left(X_{n, k}^{2}-1\right), \quad 1 \leq k \leq k_{n}, n \geq 1,
$$

Then for sufficiently large $n$ we have

$$
\begin{aligned}
\mathbf{P}\left\{Q_{n}>\lambda C_{n}^{2} / \log n\right\} & =\mathbf{P}\left\{\frac{\sum_{k=1}^{k_{n}} a_{n, k}^{2} X_{n, k}^{2}}{B_{n}^{2}}>\lambda \frac{A_{n}^{2}}{B_{n}^{2} \log n}\right\}=\mathbf{P}\left\{\sum_{k=1}^{k_{n}} a_{n, k}^{2} X_{n, k}^{2}>\lambda A_{n}^{2} \log n\right\} \\
& \leq \mathbf{P}\left\{\sum_{k=1}^{k_{n}} a_{n, k}^{2}\left(X_{n, k}^{2}-1\right)>\frac{\lambda}{2} A_{n}^{2} \log n\right\} \leq\left(\frac{\mathbf{E}\left|\sum_{k=1}^{k_{n}} Y_{n, k}\right|^{p}}{\left(\frac{\lambda}{2} A_{n}^{2} \log n\right)^{p}}\right) .
\end{aligned}
$$


Now, by Rosenthal's inequality

$$
\mathbf{E}\left|\sum_{k=1}^{k_{n}} Y_{n, k}\right|^{p} \leq\left(C_{0} \frac{p}{\log p}\right)^{p}\left\{\left|\mathbf{E}\left(\sum_{k=1}^{k_{n}} Y_{n, k}\right)^{2}\right|^{\frac{p}{2}}+\sum_{k=1}^{k_{n}} \mathbf{E}\left|Y_{n, k}\right|^{p}\right\},
$$

where $C_{0}$ is an absolute constant. But

so that

$$
\mathbf{E}\left|\sum_{k=1}^{k_{n}} Y_{n, k}\right|^{2}=\sum_{k=1}^{k_{n}} a_{n, k}^{4} \mathbf{E}\left(X_{n, k}^{2}-1\right)^{2} \leq C\|X\|_{4}^{4} \sum_{k=1}^{k_{n}} a_{n, k}^{4},
$$

$$
\begin{aligned}
\mathbf{E}\left|\sum_{k=1}^{k_{n}} Y_{n, k}\right|^{p} & \leq\left(C_{0} \frac{p}{\log p}\right)^{p}\left\{\left(C\|X\|_{4}^{4} \sum_{k=1}^{k_{n}} a_{n, k}^{4}\right)^{\frac{p}{2}}+\|X\|_{2 p}^{2 p} \sum_{k=1}^{k_{n}} a_{n, k}^{2 p}\right\} \\
& \leq C_{p} \max \left(\|X\|_{4}^{4},\|X\|_{2 p}^{2 p}\right)\left(\sum_{k=1}^{k_{n}} a_{n, k}^{4}\right)^{\frac{p}{2}}
\end{aligned}
$$

Therefore,

$$
\mathbf{P}\left\{Q_{n}>\lambda C_{n}^{2} / \log n\right\} \leq C_{p} \max \left(\|X\|_{4}^{4},\|X\|_{2 p}^{2 p}\right)\left(\frac{\left(\sum_{k=1}^{k_{n}} a_{n, k}^{4}\right)^{\frac{p}{2}}}{\left(\frac{\lambda}{2} A_{n}^{2} \log n\right)^{p}}\right) .
$$

This completes the proof of Theorem 3 .

Proof of Theorem 4. Let $Y_{1}, \ldots, Y_{n}$ be independent symmetric random variables, $S_{n}=Y_{1}+$ $\cdots+Y_{n}$. One part of the Hoffmann-Jorgensen inequality (see Hoffmann-Jorgensen (1974)) states that

$$
\mathbf{P}\left\{\left|S_{n}\right|>3^{p} t\right\} \leq C_{p} \mathbf{P}\left\{\max _{1 \leq k \leq n}\left|X_{k}\right|>t\right\}+C_{p}\left\{\mathbf{P}\left(\left|S_{n}\right|>t\right)\right\}^{2^{p}}
$$

for any integer $p \geq 1$, where $C_{p}$ is a constant depending on $p$. By (5) we have

$$
\mathbf{P}\left\{\left|T_{n}\right|>3^{p} \varepsilon A_{n}\right\} \leq D C_{p} \sum_{k=1}^{k_{n}} \mathbf{P}\left\{\left|D a_{k} X\right|>\varepsilon A_{n}\right\}+C_{p}\left(\mathbf{P}\left\{\left|T_{n}\right|>\varepsilon A_{n}\right\}\right)^{2^{p}}
$$

Choosing $p$ large enough and summing (6) for $n=1,2, \ldots$ we get

$$
\sum_{n=1}^{\infty} \mathbf{P}\left\{\left|T_{n}\right|>3^{p} \varepsilon A_{n}\right\} \leq D C_{p} \sum_{\substack{1 \leq k \leq k \\ n \geq 1}} \mathbf{P}\left\{|X|>\varepsilon A_{n} / D a_{k}\right\}+C_{p} \sum_{n=1}^{\infty}\left(\mathbf{P}\left\{\left|T_{n}\right|>\varepsilon A_{n}\right\}\right)^{2^{p}}
$$

Assumptions a) and b) therefore imply c). Conversely if c) is true, then

$$
\begin{gathered}
\mathbf{P}\left\{\left|T_{n}\right|>\varepsilon A_{n}\right\} \geq \frac{1}{2} \mathbf{P}\left\{\max _{1 \leq k \leq k_{n}}\left|a_{k} X_{k}\right| \geq \varepsilon A_{n}\right\}=\frac{1}{2}\left[1-\mathbf{P}\left\{\max _{1 \leq k \leq k_{n}}\left|a_{k} X_{k}\right|<\varepsilon A_{n}\right\}\right] \\
=\frac{1}{2}\left[1-\prod_{k=1}^{k_{n}}\left(1-\mathbf{P}\left\{\left|a_{k} X_{k}\right| \geq \varepsilon A_{n}\right\}\right)\right] \geq \frac{1}{2}\left[1-\prod_{k=1}^{k_{n}} e^{-\mathbf{P}\left\{\left|a_{k} X_{k}\right| \geq \varepsilon A_{n}\right\}}\right] \\
=\frac{1}{2}\left[1-e^{-\sum_{k=1}^{k_{n}} \mathbf{P}\left\{\left|a_{k} X_{k}\right| \geq \varepsilon A_{n}\right\}}\right]:=\frac{1}{2}\left[1-e^{-\lambda_{n}}\right] .
\end{gathered}
$$

From this estimate and c) follows that $\lambda_{n}$ tends to 0 , and then the chain of estimates can be continued as

$$
\frac{1}{2}\left[1-e^{-\lambda_{n}}\right]=\frac{1}{2}\left[\lambda_{n}+\mathcal{O}\left(\lambda_{n}^{2}\right)\right] \geq \frac{1}{4} \lambda_{n},
$$

for any integer $n$ sufficiently large. Therefore, for $n$ large

$$
\mathbf{P}\left\{\left|T_{n}\right|>\varepsilon A_{n}\right\} \geq \frac{1}{4} \lambda_{n} .
$$

And consequently c) implies $\sum_{n} \lambda_{n}<\infty$, which is exactly a). 
Proof of Theorem 5. The proof is based on a convexity argument enabling us to use the Gaussian randomization technique. First of all, there is no loss of generality in assuming that for any $n \geq 1$ and $1 \leq k \leq k_{n}$ we have

$$
\left|X_{n, k}\right| \leq 1 \quad \text { a.s. }
$$

Let $\mathcal{X}^{\prime}$ be an independent copy of $\mathcal{X}$ defined on a joint probability space $\left(\Omega^{\prime}, \mathcal{A}^{\prime}, \mathbf{P}^{\prime}\right)$ with corresponding expectation symbol $\mathbf{E}^{\prime}$. Write $T_{n}^{\prime}=\sum_{k=1}^{k_{n}} a_{n, k} X_{n, k}^{\prime}$. Let $\varepsilon=\left\{\varepsilon_{n, k}, 1 \leq k \leq k_{n}, n \geq 1\right\}$ be a triangular array of independent Rademacher random variables defined on a joint probability space $\left(\Omega_{\varepsilon}, \mathcal{A}_{\varepsilon}, \mathbf{P}_{\varepsilon}\right)$, with corresponding expectation symbol $\mathbf{E}_{\varepsilon}$. Similarly, let $\mathbf{g}=\left\{g_{n, k}, 1 \leq k \leq\right.$ $\left.k_{n}, n \geq 1\right\}$ be a triangular array of independent $\mathcal{N}(0,1)$ distributed random variables defined on a joint probability space $\left(\Omega_{g}, \mathcal{A}_{g}, \mathbf{P}_{g}\right)$, with corresponding expectation symbol $\mathbf{E}_{g}$. Let $A$ be any real number and consider the convex non-decreasing function $\varphi_{A}(x)=(x-A)^{+}$. If $X$ is any random variable, then for any positive real $a, a \mathbf{P}\{X>A+a\} \leq \mathbf{E} \varphi_{A}(X)$. Applying this for $A=A_{n} \varepsilon=a$ and $X=T_{n}$ and then using Jensen's inequality lead to

$$
\begin{aligned}
& \left(\varepsilon A_{n}\right) \mathbf{P}\left\{T_{n}>2 \varepsilon A_{n}\right\} \leq \mathbf{E} \varphi_{\varepsilon A_{n}}\left(T_{n}\right)=\mathbf{E} \varphi_{\varepsilon A_{n}}\left(T_{n}-\mathbf{E}^{\prime} T_{n}^{\prime}\right) \\
& \leq \mathbf{E} \mathbf{E}^{\prime} \varphi_{\varepsilon A_{n}}\left(T_{n}-T_{n}^{\prime}\right)=\mathbf{E} \mathbf{E}_{\varepsilon} \varphi_{\varepsilon A_{n}}\left(\sum_{k=1}^{k_{n}} a_{n, k} \varepsilon_{n, k} X_{n, k}\right) \\
& =\mathbf{E} \mathbf{E}_{\varepsilon} \varphi_{\varepsilon A_{n}}\left(\frac{\sum_{k=1}^{k_{n}} a_{n, k} \varepsilon_{n, k}\left(\mathbf{E}_{g}\left|g_{n, k}\right|\right) X_{n, k}}{(2 / \pi)^{1 / 2}}\right) \\
& \leq \mathbf{E} \mathbf{E}_{\varepsilon} \mathbf{E}_{g} \varphi_{\varepsilon A_{n}}\left(\frac{\sum_{k=1}^{k_{n}} a_{n, k} \varepsilon_{n, k}\left|g_{n, k}\right| X_{n, k}}{(2 / \pi)^{1 / 2}}\right) \\
& =\mathbf{E} \mathbf{E}_{g} \varphi_{\varepsilon A_{n}}\left(\frac{\sum_{k=1}^{k_{n}} a_{n, k} g_{n, k} X_{n, k}}{(2 / \pi)^{1 / 2}}\right) .
\end{aligned}
$$

In the last equality we used the fact that $\left\{\varepsilon_{n, k}\left|g_{n, k}\right|, 1 \leq k \leq k_{n}, n \geq 1\right\} \stackrel{\mathcal{D}}{=}\left\{g_{n, k}, 1 \leq k \leq\right.$ $\left.k_{n}, n \geq 1\right\}$. Applying it now to $A=A_{n} \varepsilon=a$ and $X=-T_{n}$, and arguing similarly also gives

$$
\left(\varepsilon A_{n}\right) \mathbf{P}\left\{-T_{n}>2 \varepsilon A_{n}\right\} \leq \mathbf{E} \mathbf{E}_{g} \varphi_{\varepsilon A_{n}}\left(\frac{\sum_{k=1}^{k_{n}} a_{n, k} g_{n, k} X_{n, k}}{(2 / \pi)^{1 / 2}}\right) .
$$

As $\mathbf{P}\left\{\left|T_{n}\right|>2 \varepsilon A_{n}\right\} \leq \mathbf{P}\left\{T_{n}>2 \varepsilon A_{n}\right\}+\mathbf{P}\left\{-T_{n}>2 \varepsilon A_{n}\right\}$, we obtain from (7) and (8)

$$
\left(\varepsilon A_{n}\right) \mathbf{P}\left\{\left|T_{n}\right|>2 \varepsilon A_{n}\right\} \leq 2 \mathbf{E} \mathbf{E}_{g} \varphi_{\varepsilon A_{n}}\left(\frac{\sum_{k=1}^{k_{n}} a_{n, k} g_{n, k} X_{n, k}}{(2 / \pi)^{1 / 2}}\right) .
$$

But,

$$
\begin{aligned}
\mathbf{E}_{g} \varphi_{\varepsilon A_{n}}\left(\frac{\sum_{k=1}^{k_{n}} a_{n, k} g_{n, k} X_{n, k}}{(2 / \pi)^{1 / 2}}\right) & =\int_{\varepsilon A_{n}}^{\infty} \mathbf{P}\left\{\mathcal{N}(0,1)>\frac{(2 / \pi)^{1 / 2} u}{V_{n}}\right\} d u \\
& =\frac{V_{n}}{\sqrt{2 / \pi}} \int_{\frac{\sqrt{2 / \pi} \varepsilon A_{n}}{V_{n}}}^{\infty} \mathbf{P}\{\mathcal{N}(0,1)>v\} d v \\
& =\frac{V_{n}}{\sqrt{2 / \pi}} \int_{\frac{\sqrt{2 / \pi} \varepsilon A_{n}}{V_{n}}}^{\infty} \int_{v}^{\infty} e^{-w^{2} / 2} \frac{d w}{\sqrt{2 \pi}} d v \\
& =\frac{V_{n}}{2} \int_{\frac{\sqrt{2 / \pi} \varepsilon A_{n}}{T_{n}}}^{\infty} R(v) e^{-v^{2} / 2} d v \leq \sqrt{\frac{\pi}{2}} \frac{V_{n}}{2} R\left(\frac{\sqrt{2 / \pi} \varepsilon A_{n}}{V_{n}}\right) e^{-\frac{\varepsilon^{2} A_{n}^{2}}{\pi V_{n}^{2}}} \\
& \leq \frac{\pi V_{n}}{4} e^{-\frac{\varepsilon^{2} A_{n}^{2}}{\pi V_{n}^{2}}} .
\end{aligned}
$$


Therefore

$$
\mathbf{P}\left\{\left|T_{n}\right|>2 \varepsilon A_{n}\right\} \leq \mathbf{E} \frac{\pi V_{n}}{4 \varepsilon A_{n}} e^{-\frac{\varepsilon^{2} A_{n}^{2}}{\pi V_{n}^{2}}} .
$$

We now make use of the boundedness assumption on the sequence $\mathcal{X}$. The above inequality becomes in this case

$$
\mathbf{P}\left\{\left|T_{n}\right|>2 \varepsilon A_{n}\right\} \leq \frac{\pi}{4 \varepsilon} \mathbf{E} e^{-\frac{\varepsilon^{2} A_{n}^{2}}{\pi V_{n}^{2}}},
$$

since $V_{n}^{2}=\sum_{k=1}^{k_{n}} a_{n, k}^{2} X_{n, k}^{2} \leq \sum_{k=1}^{k_{n}} a_{n, k}^{2} \leq A_{n}^{2}$ a.s. Put for $m=1, \ldots$

$$
J_{m}=\left\{n: m \leq A_{n} / V_{n}<m+1\right\}
$$

Then

$$
\begin{aligned}
\sum_{n=1}^{\infty} \mathbf{P}\left\{\left|T_{n}\right|>2 \varepsilon A_{n}\right\} & \leq \frac{\pi}{4 \varepsilon} \sum_{m=1}^{\infty} \mathbf{E} \sum_{n \in J_{m}} e^{-\frac{\varepsilon^{2} A_{n}^{2}}{\pi V_{n}^{2}}} \leq \frac{\pi}{4 \varepsilon} \sum_{m=1}^{\infty} \mathbf{E}\left[\sharp\left\{J_{m}\right\} e^{-\frac{\varepsilon^{2} m^{2}}{2 \pi}}\right] e^{\frac{\varepsilon^{2} m^{2}}{2 \pi}-\frac{\varepsilon^{2} m^{2}}{\pi}} \\
& \leq \frac{\pi}{4 \varepsilon} \mathbf{E} \sup _{m \geq 1}\left[\sharp\left\{J_{m}\right\} e^{-\frac{\varepsilon^{2} m^{2}}{2 \pi}}\right]\left[\sum_{m=1}^{\infty} e^{-\frac{\varepsilon^{2} m^{2}}{2 \pi}}\right] \\
& \leq C_{\varepsilon} \mathbf{E} \sup _{m \geq 1} \frac{\sharp\left\{n: m<A_{n} / V_{n} \leq m+1\right\}}{\exp \left\{\varepsilon^{2} m^{2} / 2 \pi\right\}} .
\end{aligned}
$$

This completes the proof of Theorem 5 .

Acknowledgment. We thank Anna Kuczmaszewska for several useful remarks concerning the first draft of the paper.

\section{References}

1. Adler A., Cabrera, M., Rosalsky A., Volodin A. [1999] Degenerate weak convergence of row sums fors arrays of random elements in stable type $p$ Banach spaces, Bull. Inst. Math. Acad. Sinica 27, 187-212.

2. Ahmed S., Antonini R.G., Volodin A. [2002] On the rate of complete convergence for weighted sums of arrays of Banach space valued random elements with application to moving average processes, Statist. Probab. Lett. 58, 185-194.

3. Baum L.E., Katz M. [1965] Convergence rates in the law of large numbers, Trans. Amer. Math. Soc. 120, 108-123.

4. Breiman L. [1968] Probability, Addison-Wesley.

5. Csörgö M., Révész P. [1981] Strong Approximations in Probability and Statistics, Academic Press.

6. Davis, B. [1976] On the $L_{p}$ norms of stochastic integrals and other martingales, Duke Math. J. 43, 697-704.

7. Erdős P. [1949] On a theorem of Hsu and Robbins, Ann. Math. Stat. 20, 286-291.

8. Fazekas I. [1985] Convergence rates in the Marczinkiewicz strong law of large numbers for Banach space values random variables with multidimensional indices, Publ. Math. Debrecen 32, 203-209.

9. Fazekas I. [1992] Convergence rates in the law of large numbers for arrays, Publ. Math. Debrecen 41, 53-71. 
10. Fisher E. [1992] A Skorohod representation and an invariance principle for sums of weighted i.i.d. random variables, Rocky Mount. J. Math. 22, 169-179.

11. Gut A. [1992] Complete convergence for arrays, Period. Math. Hungar. 25, 51-75.

12. Hoffmann-Jorgensen J. [1974] Sums of independent valued Banach spaces random variables, Studia Math. 52, 159-186.

13. Hu T.-C., Móricz F., Taylor R.L. [1989] Strong laws of large numbers for arrays of rowwise independent random variables, Acta Math. Hungar. 54, 153-162.

14. Hu T.-C., Rosalsky A., Szynal D., Volodin A. [1999] On complete convergence for arrays of rowwise independent random elements in Banach spaces, Stoch. Anal. Appl. 17, 963-992.

15. Hsu P.L., Robbins H. [1947] Complete convergence and the law of large numbers, Proc. Nat. Acad. Sci. USA 33, 25-31.

16. Kuczmaszewska A., Szynal D. [1988] On the Hsu-Robbins law of large numbers for subsequences, Bull. Polish Acad. Sci. Math. 36, 69-79.

17. Kuczmaszewska A., Szynal D. [1990] On complete convergence for partial sums of independent identically distributed random variables, Prob. Math. Statist. 11, 223-235.

18. Kuczmaszewska A., Szynal D. [1994] On complete convergence in a Banach space, Internat. J. Math. Math. Sci. 17, 1-14.

19. Li D., Rao M.B., Wang X. [1992] Complete convergence of moving average processes, Statist. Probab. Lett. 14, 111-114.

20. Mitrinović D. S. [1970] Analytic inequalities, Die Grundlehren der mathematischen Wissenschaften, Band 1965, Springer-Verlag.

21. Petrov, V.V. [1995] Limit theorems of probability theory. Sequences of independent random variables, Clarendon Press, Oxford.

22. Pruitt, W. [1966] Summability of independent random variables, J. Math. Mech. 15 769776.

23. Rao M.B., Wang X., Yang X. [1993] Convergence rates on strong laws of large numbers for arrays of rowwise independent elements, Stoch. Anal. Appl. 11, 115-132.

24. Rohatgi V.K. [1971] Convergence of weighted sums of independent random variables, Proc. Cambridge Philos. Soc. 69, 305-307.

25. Sung S.H. [1997] Complete convergence for weighted sums of arrays of rowwise independent $B$-valued random variables, Stoch. Anal. Appl. 15, 255-267.

26. Weber M. [1995] Borel matrix, Comment. Math. Univ. Carolin. 36, 401-415.

27. Weber M. [2006] A weighted CLT, Stat. Prob. Letters 76, 1482-1487.

Istvan Berkes, Technical University Graz, Department of Statistics, Steyrergasse 17/IV, A-8010 Graz, Austria. E-MAIL: berkes@tugraz.at

Michel Weber: Mathématique (IRMA), Université Louis-Pasteur et C.N.R.S., 7 rue René Descartes, 67084 Strasbourg Cedex, France. E-mail: weber@math.u-strasbg.fr 